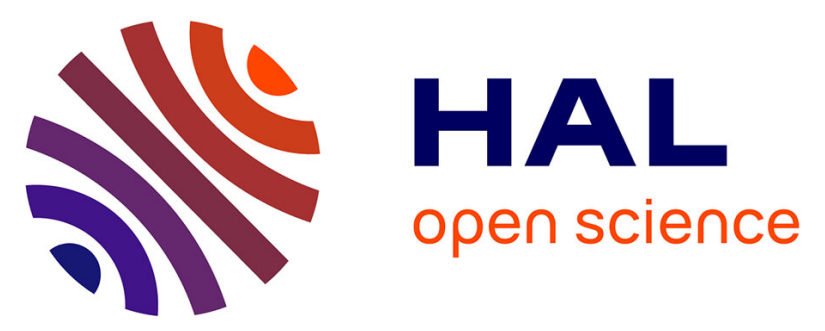

\title{
Ponderomotive effects in the femtosecond plasmon-assisted photoelectric effect in bulk metals: Evidence for coupling between surface and interface plasmons
}

Michèle Raynaud, Joseph Kupersztych

\section{To cite this version:}

Michèle Raynaud, Joseph Kupersztych. Ponderomotive effects in the femtosecond plasmon-assisted photoelectric effect in bulk metals: Evidence for coupling between surface and interface plasmons. Physical Review B: Condensed Matter and Materials Physics (1998-2015), 2007, 10.1103/PhysRevB.76.241402 . hal-01327436

\section{HAL Id: hal-01327436 \\ https://hal.science/hal-01327436}

Submitted on 8 Jun 2016

HAL is a multi-disciplinary open access archive for the deposit and dissemination of scientific research documents, whether they are published or not. The documents may come from teaching and research institutions in France or abroad, or from public or private research centers.
L'archive ouverte pluridisciplinaire HAL, est destinée au dépôt et à la diffusion de documents scientifiques de niveau recherche, publiés ou non, émanant des établissements d'enseignement et de recherche français ou étrangers, des laboratoires publics ou privés. 


\title{
Ponderomotive effects in the femtosecond plasmon-assisted photoelectric effect in bulk metals: Evidence for coupling between surface and interface plasmons
}

\author{
Michèle Raynaud* and Joseph Kupersztych \\ SPCSI, CEA/Saclay, 91191 Gif-sur-Yvette, France \\ (Received 18 October 2007; published 12 December 2007)
}

\begin{abstract}
The existence of ponderomotive acceleration effects in the metal bulk has been experimentally demonstrated in the context of the femtosecond plasmon-assisted multiphoton photoelectric effect in metal systems. The resulting electron energy spectra show that these effects essentially depend on the coupling between the surface and interface plasmons. While the essential part played by the ponderomotive force of the surface plasmon is to accelerate the photoelectrons in the vacuum, the dramatic enhancement of the photoelectron production and the angular dependence in the photoemission process mainly result from ponderomotive effects in the metal bulk.
\end{abstract}

DOI: 10.1103/PhysRevB.76.241402

PACS number(s): 78.70.-g, 73.20.Mf, 78.40.Kc, 79.60.-i

In the photoelectric effect in metals, photoelectrons may be emitted from a region near the metal surface ${ }^{1,2}$ or may originate from the bulk. ${ }^{3}$ In the bulk, electron excitation occurs via photon absorption in the presence of a third body (ion or impurity) or through interband transitions. On the other hand, the surface effects play an essential role for the number of electrons emitted while the absorption of light energy in the surface layer (a few nanometers) is relatively small (about $0.1 \%$ of the total absorption rate). In recent years, much attention has been paid to the possibility of enhancing electron emission of metals through the surface photoelectric effect by means of light excitation of electron collective oscillation modes localized near the metal surface. ${ }^{4}$ Recently, it has been shown that the photoelectrons emerging from a metal via a multiphoton photoelectric effect in the presence of femtosecond laser-excited surface plasmons are much more energetic than in conventional photoemission. ${ }^{5-9}$ The phenomenon has been interpreted in terms of ponderomotive acceleration of the photoelectrons in the vacuum by the strongly inhomogeneous high-frequency electric field of the surface plasmon surrounding the metal surface. ${ }^{5}$

However, ponderomotive effects are not restricted to those electrons that have been released out of the metal. The field of the surface plasmon is also strongly inhomogeneous in the metal due to its small spatial extension. For example, by using a simple dielectric description, ${ }^{10}$ the value $\mathcal{D}$ of the depth at which the field amplitude of the surface plasmon falls to $1 / e$, for a metal like gold and for a laser wavelength of $800 \mathrm{~nm}$, is readily obtained as $\mathcal{D}_{M} \simeq 22 \mathrm{~nm}$ in the metal and $\mathcal{D}_{V} \simeq 650 \mathrm{~nm}$ in the vacuum. The ponderomotive force $F_{p} \simeq U_{p} / \mathcal{D}$ (where $U_{p}$ denotes the ponderomotive potential of the surface plasmon) is thus 30 times stronger in the metal than in the vacuum. In this paper, we will show that, under certain conditions, inner-metal ponderomotive acceleration effects exist and that they can play an essential part in the photoemission process in the presence of resonant electron collective excitations.

To this end, let us consider a bilayer metal structure such as a thin gold overlayer of thickness $\mathcal{L}$ deposited on a massive aluminum sample, the surface of which is periodically structured to form a grating surface. For an optimum overlayer thickness $\mathcal{L}_{\text {opt }}$, the field of the laser-excited surface plasmon can tunnel through the overlayer and excites electron density fluctuations (interface plasmons) at the interface between the two metals. ${ }^{11}$ Arguing by analogy with the phenomenon of ponderomotive acceleration of the electron in vacuum by the field of the surface plasmon, ponderomotive acceleration of the conduction electrons by the strongly inhomogeneous field of the interface plasmon can occur, so that energy and momentum can be transferred from the interface plasmon to the electrons in the bulk of the overlayer. The existence of such a phenomenon was invoked to explain the reduction of the number $N$ of photons needed to be absorbed by each photoelectron in the multiphoton photoelectric process and the corresponding increasing of the photoemission probability by a factor greater than $10^{4}$ in comparison with a homogeneous metal structure. ${ }^{12}$

It should be remarked that the energy transfer from the surface plasmon to the electrons in the metal bulk is a mechanism that manifests itself through significant modifications in the photoemission process itself. As some part of the electromagnetic energy of the surface plasmon is pumped by the field of the interface plasmon to drive electron density fluctuations at the metal-metal interface, the magnitude of the interface plasmon is expected to increase at the expense of a depletion of the surface plasmon amplitude. As a consequence, the ponderomotive force experienced by the photoelectrons in the evanescence length of the surface plasmon will decrease, so that their kinetic energy will decrease accordingly. Therefore, experimental evidence for the coupling between surface and interface plasmons can be obtained by comparing the electron energy spectra resulting from different metal structures, depending on whether the conditions for interface plasmon excitation exist or not.

In order to investigate the part played by inner-metal ponderomotive acceleration effects, we have performed a systematic study of the electron energy spectra together with the angular variations of the photoemission. We used a magnetically shielded time-of-flight spectrometer of length $60 \mathrm{~cm}$. The surfaces of the targets were located at $0.7 \mathrm{~cm}$ from the spectrometer, the acceptance angle of which was smaller than $2^{\circ}$. The energy resolution was less than $0.1 \mathrm{eV}$ for a flight potential of $2 \mathrm{~V}$. For these values and in the range of the detected photoelectron signals, space charge effects were 


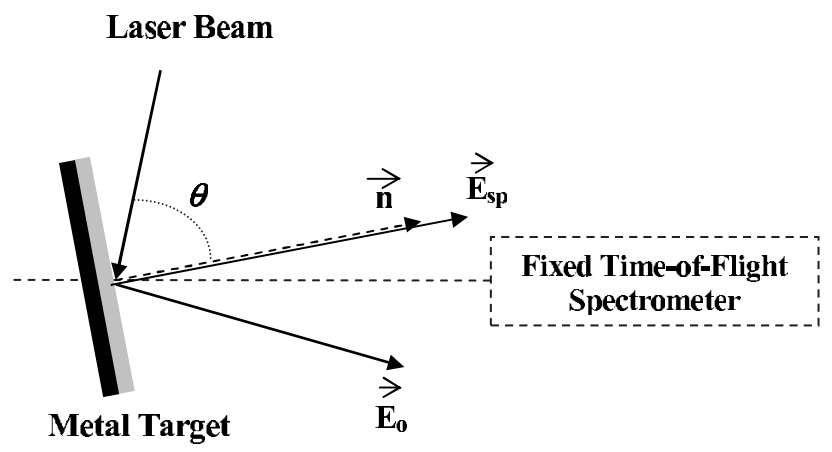

FIG. 1. Schematic representation of the angular detection of the photoelectrons. The target can be moved to vary the incidence angle $\theta$ of the laser and so that the normal $\vec{n}$ to the surface lies within the detection angle of the spectrometer.

verified to be negligible. The principle of these experiments is schematically described on Fig. 1.

To determine the optimum conditions for the mechanism of energy transfer to the conduction electrons, we first performed angle-integrated experiments similar to those previously described ${ }^{12}$ on a series of metal gratings made of gold overlayers with thicknesses $\mathcal{L}$ from 18 to $200 \mathrm{~nm}$ deposited on a massive aluminum sample. The wavelength of the $p$-polarized laser beam was $800 \mathrm{~nm}$, so that four photons of energy $1.55 \mathrm{eV}$ need to be absorbed per electron, as required by the multiphoton photoelectric equation (polycrystalline gold work function $W_{\mathrm{Au}}=5.1 \mathrm{eV}$ ). The optimum electron signal was still obtained with a sample with gold overlayer of thickness near $\mathcal{L}_{\text {opt }} \simeq 43 \mathrm{~nm}$ for the resonance angle $\theta_{\text {res }}$ $=67^{\circ}$. For this optimum thickness, as was already shown in Ref. 12, the number of absorbed photons per electron in the photoemission process, characterized by the variations of the photoelectron current versus laser intensity, for intensities as low as $I=0.1 \mathrm{GW} / \mathrm{cm}^{2}$, was 3 instead of 4 . In fact, this result reveals that the conduction electrons in the metal overlayer have gained some additional energy due to the presence of the electron collective excitation, so that they need to absorb a lower number of photons to overcome the potential barrier at the metal surface. Hence, in keeping with the above discussion, this effect is expected to be accompanied by a simultaneous decrease of the photoelectron energy due to the corresponding depletion of the surface plasmon energy. In other words, for equal laser intensities and pulse durations, the energy of the photoelectrons is expected to be lower in the case of the optimum bilayer emissive sample than in the case of the ultrathick gold sample, where interface plasmon excitation cannot occur.

The electron energy spectra shown in Fig. 2 are quite significant inasmuch as they reveal, as expected, that the photoelectrons emitted by the most emissive sample $\left(\mathcal{L}_{\text {opt }}\right.$ $\simeq 43 \mathrm{~nm}$ ) are much less energetic than those produced by the least emissive (bulk) gold sample $(\mathcal{L}=200 \mathrm{~nm})$ and the thinner bilayer sample $(\mathcal{L}=28 \mathrm{~nm})$. Hence, for equal laser intensities, the ponderomotive force of the surface plasmon is effectively weaker in the case of the optimum emissive bimetal sample than in the case of the bulk gold sample, where an interface plasmon cannot exist. This result clearly shows

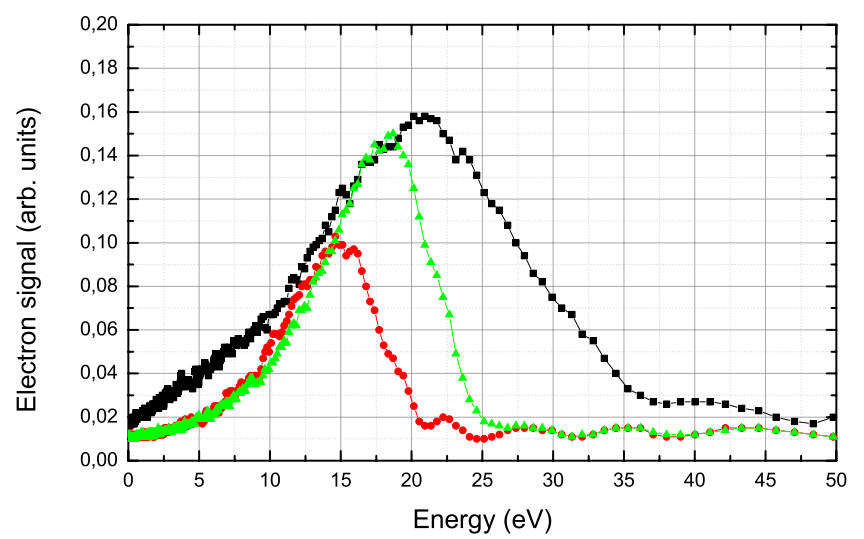

FIG. 2. (Color online) Photoelectron energy spectra for three typical samples with gold overlayer thicknesses $200(\boldsymbol{\square}), 28(\boldsymbol{\Delta})$, and $43 \mathrm{~nm}(\bullet)$. The laser intensity was $I=4 \mathrm{GW} / \mathrm{cm}^{2}$ and the pulse duration $200 \mathrm{fs}$.

evidence for the coupling between the surface and interface plasmons.

Furthermore, another piece of evidence for this coupling has been obtained by investigating the electron response of the various bilayer samples at different laser intensities. As a matter of fact, when the surface plasmon is coupled to the field of the interface plasmon, as is the case for the most emissive sample, its energy is optimally transferred to the interface plasmon and, because of this dissipation of the surface plasmon energy, the dependence of the photoelectron energy on the laser intensity is no longer linear. Thus, a nonlinear electron response of the bilayer samples can be evidenced by measuring the electron energy spectra at different laser intensities. In Fig. 3, the spectra obtained by simply doubling the value of the laser intensity are represented. It clearly appears that the position of the peaks corresponding to gold overlayer thicknesses of 28 and $43 \mathrm{~nm}$ does not vary linearly, with the laser intensity, in contrast with the peak corresponding to an overlayer thickness of $200 \mathrm{~nm}$. It can therefore be concluded that the electron response is not linear when interface plasmon excitation can occur and is linear when only a surface plasmon is excited.

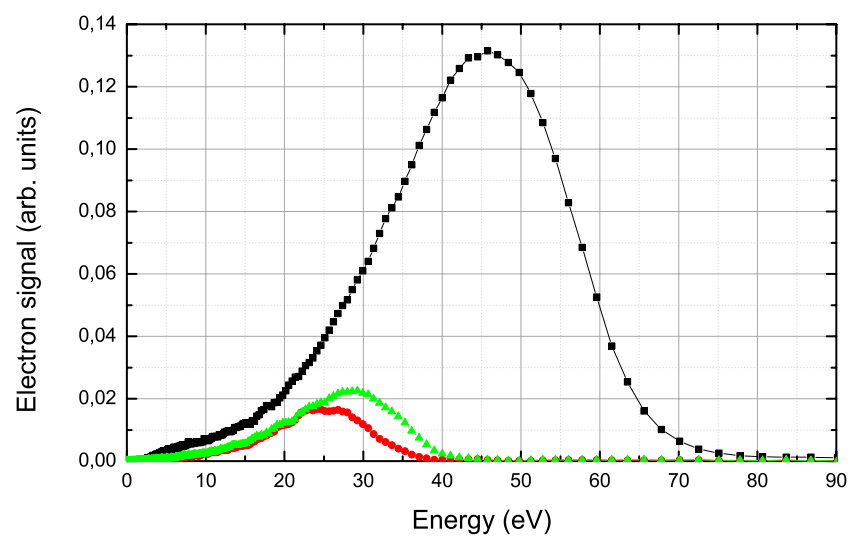

FIG. 3. (Color online) Same as in Fig. 2 but for $I=8 \mathrm{GW} / \mathrm{cm}^{2}$. 


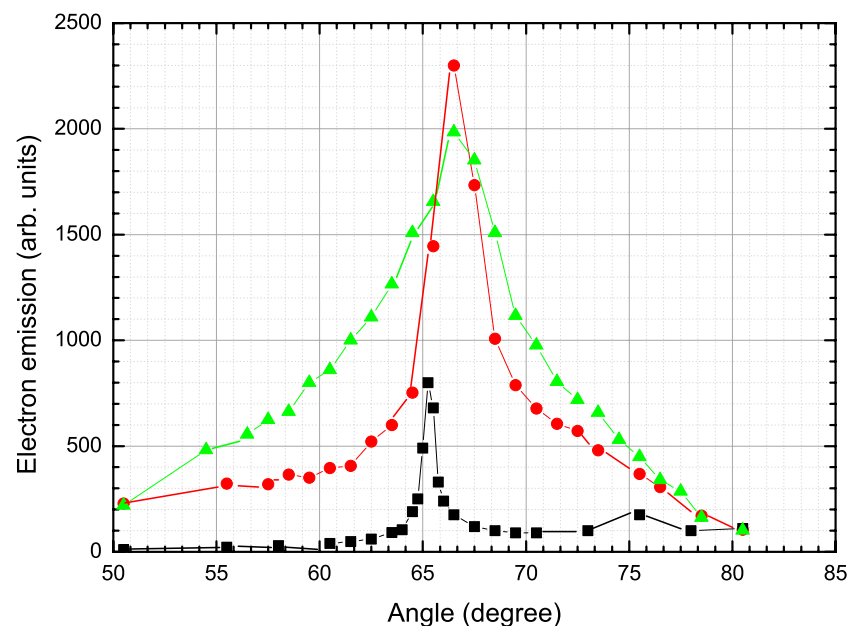

FIG. 4. (Color online) Plasmon resonance angles for three samples of overlayer thicknesses: $200 \mathrm{~nm}(\boldsymbol{\square}), 43 \mathrm{~nm}(\bullet)$, and $28 \mathrm{~nm}(\boldsymbol{\Delta})$. The laser intensities were, respectively, 1, 0.1, and $0.15 \mathrm{GW} / \mathrm{cm}^{2}$, and the pulse durations $50 \mathrm{fs}$.

An estimate of the maximum energy $\Delta \mathcal{E}$ that can be gained by the conduction electrons through ponderomotive acceleration can be obtained as follows. The final kinetic energy $\mathcal{E}$ of a freed electron of initial kinetic energy $\mathcal{E}_{i}$ in a high-frequency plasmon field (in the case of full conversion of the ponderomotive potential ${ }^{13}$ ) can be easily obtained as

$$
\mathcal{E}(\phi)=U_{p}+2 U_{p}\left[\left(\mathcal{E}_{i} / 2 U_{p}\right)^{1 / 2}-\cos \phi\right]^{2},
$$

where $U_{p}=e^{2} \eta^{2} E_{0}^{2} / 4 m \omega_{0}^{2}$ denotes the ponderomotive potential of the plasmon, $E_{0}$ is the amplitude of the laser field of angular frequency $\omega_{0}, \eta(\tau)$ is the resonance factor for an effective plasmon lifetime $\tau$, and $\phi$ is the phase seen by the electron at the instant of its release. The final energy of the photoelectron thus depends both on the value of the ponderomotive potential and on the initial phase experienced by the freed electron. ${ }^{5,13}$ In particular, the main peak in the electron energy spectrum provides the value of the ponderomotive potential of the surface plasmon via the simple relation

$$
\langle\mathcal{E}\rangle_{\phi}=2 U_{s p}\left(1+\mathcal{E}_{i} / 2 U_{s p}\right),
$$

while its broadening results from the random phase of the plasmon field experienced by the released electrons. An immediate consequence shown by Eq. (2) is that, in the resonant case $\left(U_{s p} \gg \mathcal{E}_{i}\right)$, the value of the main electron energy peak varies linearly with the ponderomotive potential of the surface plasmon, that is, the laser intensity, a property that is exemplified in Figs. 2 and 3 through the electron response of the ultrathick gold sample. The value of the ponderomotive potential $U_{s p}$ of the surface plasmon, for a given laser intensity, can thus be obtained from Eq. (2) and from the measured values of the energy of the photoelectrons emitted by the target. On the other hand, if $\alpha$ denotes the fraction of the surface plasmon energy transferred to the interface plasmon via the tunneling effect, conservation of energy can be expressed as

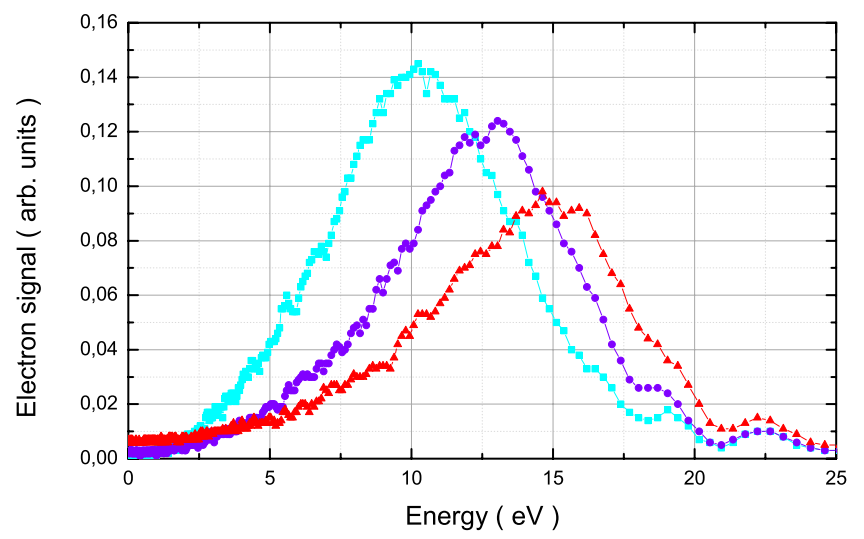

FIG. 5. (Color online) Photoelectron energy spectra produced by the most emissive sample (overlayer thickness $43 \mathrm{~nm}$ ) for three laser incidence angles: $\theta=\theta_{\text {res }}=67^{\circ}(\mathbf{\Delta}), \theta=69^{\circ}(\bullet)$, and $\theta=74^{\circ}$ (ם). The laser intensity was $I=4 \mathrm{GW} / \mathrm{cm}^{2}$ and the pulse duration $200 \mathrm{fs}$.

$$
\left(\mathcal{D}_{M}+\mathcal{D}_{V}\right) \alpha U_{s p} \simeq 2 \mathcal{D}_{M} U_{i p},
$$

where $U_{i p}$ denotes the ponderomotive potential of the interface plasmon. The order of magnitude of the maximum energy $\Delta \mathcal{E}$ that can be gained by the conduction electrons is then readily obtained via Eqs. (2) and (3). For example, for a laser intensity $I=8 \mathrm{GW} / \mathrm{cm}^{2}$, Fig. 3 gives $U_{s p}(8) \simeq 23 \mathrm{eV}$. Thus, for the same ultrathick gold sample, using the above linearity property, the value of the ponderomotive potential of the surface plasmon for a laser intensity $I=0.1 \mathrm{GW} / \mathrm{cm}^{2}$ is $U_{s p}(0.1) \simeq 0.28 \mathrm{eV}$. It follows that, with $\mathcal{D}_{M} \simeq 22 \mathrm{~nm}$ and $\mathcal{D}_{V} \simeq 650 \mathrm{~nm}$, the value of the ponderomotive potential of the interface plasmon is, in $\mathrm{eV}$, approximately $U_{i p}(0.1)$ $\simeq 4.3 \alpha$, and the maximum energy that can be acquired by the conduction electrons is then given by Eq. (2) with ponderomotive potential $U_{i p}$ as $\Delta \mathcal{E}(0.1) \simeq 8.6 \alpha$. Hence, for a laser intensity near $I=0.1 \mathrm{GW} / \mathrm{cm}^{2}$, it appears that only three photons of energy $1.55 \mathrm{eV}$ instead of four need to be absorbed by the energetic conduction electrons to overcome the gold work function $W_{\mathrm{Au}}=5.1 \mathrm{eV}$, as shown in Fig. 3 of Ref. 12, provided the fraction of energy transferred from the surface plasmon to the interface plasmon is at least of the order of $5 \%$. This phenomenon is likely to occur for the sample with an optimum overlayer thickness $\left(\mathcal{L}_{\text {opt }} \simeq 43 \mathrm{~nm}\right)$. For less emissive samples, such as thinner bilayer samples of thickness $\mathcal{L}=28$ or $18 \mathrm{~nm}$, the coupling parameter $\alpha$ is smaller, so that the corresponding changes of slope ${ }^{12}$ require higher values of the ponderomotive potential, that is, higher laser intensities.

On the other hand, it is worth noting that ponderomotive effects in the metal bulk play also an essential part in the angular dependence of the photoemission process in the presence of electron collective excitation. As the fields of the surface and interface plasmons are essentially directed along the normal to the metal surface, the corresponding ponderomotive forces, which are also directed along the normal to the surface, tend to deviate the electrons in the same direction. Hence, the photoelectrons that would be mainly emitted 
along the direction of polarization of the laser field in the absence of electron collective excitation are deviated toward the normal to the metal surface via a ponderomotive acceleration effect and are not collected in the spectrometer. This explains why, in apparent contradiction with the angleintegrated measurements, the electron current emitted by the most emissive sample is much lower (see Figs. 2 and 3) than those given by the samples of overlayer thicknesses $\mathcal{L}=200$ and $28 \mathrm{~nm}$.

To more precisely explore the angular dependence of the photoemission, we have employed a method that takes advantage of the broadening (due to the coupling between the two opposite faces of the overlayer) of the surface plasmon resonance angle of the bilayer metal samples (see Fig. 4). By simply rotating the metal sample (see Fig. 1), we could easily vary the incidence angle of the laser beam within the broadening range, keeping in mind the fact that the magnitude of the surface plasmon decreases correspondingly. The resulting energy spectra displayed in Fig. 5 show, as expected, that the more the incidence angle of the laser differs from the optimum plasmon resonance angle $\left(\theta_{\text {res }}=67^{\circ}\right)$, the more the energy of the photoelectrons is reduced due to the decrease of the ponderomotive force in the vacuum. On the other hand, when the angle of incidence is close to the value $\theta_{\perp}=74^{\circ}$, for which the perpendicular to the metal surface just coincides with the axis of the spectrometer, the detected photoelectron signal becomes maximum. We can therefore conclude that the photoelectrons resulting from the most emissive sample are essentially emitted perpendicularly to the metal surface and that this angular dependence is mainly due to a ponderomotive effect in the metal bulk.

In conclusion, enhanced photoemission in the presence of laser-excited surface and interface plasmons can be interpreted as a Spicer-like three-step process. ${ }^{3}$ In the first step, the electrons in the metal bulk are accelerated toward the surface by the ponderomotive force of the interface plasmon. The second process is a quantum absorption phenomenon in which the energetic conduction electrons absorb a number of photons lower than the minimum number required by the multiphoton photoelectric equation to overcome the work function of the metal. ${ }^{12}$ Finally, the released electrons can gain a large amount of energy because of their acceleration in the vacuum by the ponderomotive force of the surface plasmon. ${ }^{5-8}$

We gratefully acknowledge the technical staff of the LUCA Saclay Laser Facility for their help in the experiments.
*Present address: LSI, CEA/Saclay, 91191 Gif-sur-Yvette, France.

${ }^{1}$ I. Adawi, Phys. Rev. 134, A788 (1964).

${ }^{2}$ B. Feuerbacher and B. Fitton, Phys. Rev. Lett. 29, 786 (1972); B.

J. Waclawski and E. Plummer, ibid. 29, 783 (1972).

${ }^{3}$ H. Y. Fan, Phys. Rev. 68, 43 (1945); W. E. Spicer, ibid. 112, 114 (1958).

${ }^{4}$ J. Hofmann and W. Steinmann, Phys. Status Solidi 30, K53 (1968); J. Endriz and W. Spicer, Phys. Rev. Lett. 24, 64 (1970); W. H. Weber and G. W. Ford, Opt. Lett. 6, 122 (1981); J. T. Stuckless and M. Moskovits, Phys. Rev. B 40, 9997 (1989); T. Tsang, T. Srinivasan-Rao, and J. Fischer, ibid. 43, 8870 (1991); M. Aeschlimann, C. A. Schmuttenmaer, H. E. Elsayed-Ali, R. J. D. Miller, J. Cao, Y. Gao, and D. A. Mantell, J. Chem. Phys. 102, 8606 (1995); V. M. Shalaev, C. Douketis, T. Haslett, T. Stuckless, and M. Moskovits, Phys. Rev. B 53, 11193 (1996).

${ }^{5}$ J. Kupersztych, P. Monchicourt, and M. Raynaud, Phys. Rev. Lett. 86, 5180 (2001).

${ }^{6}$ J. Zawadzka et al., Appl. Phys. Lett. 79, 2130 (2001).
${ }^{7}$ S. E. Irvine, A. Dechant, and A. Y. Elezzabi, Phys. Rev. Lett. 93, 184801 (2004).

${ }^{8}$ G. H. Welsh, N. T. Hunt, and K. Wynne, Phys. Rev. Lett. 98, 026803 (2007).

${ }^{9}$ The acceleration effect has been recently extrapolated to the attosecond regime by M. I. Stockman, M. F. Kling, U. Kleineberg, and F. Krausz, Nat. Photonics 1, 539 (2007). The high-intensity regime has been recently investigated by M. Raynaud, J. Kupersztych, C. Riconda, J. C. Adam, and A. Héron, Phys. Plasmas 14, 092702 (2007).

${ }^{10}$ H. Raether, Surface Plasmons on Smooth and Rough Surfaces and on Gratings (Springer-Verlag, Berlin, 1988).

${ }^{11}$ E. A. Stern and R. A. Ferrel, Phys. Rev. 120, 130 (1960); J. Kupersztych and M. Raynaud, Solid State Commun. 106, 785 (1998).

${ }^{12}$ J. Kupersztych and M. Raynaud, Phys. Rev. Lett. 95, 147401 (2005).

${ }^{13}$ J. Kupersztych, Phys. Scr. 42, 51 (1990). 